CLINICAL STUDY

\title{
Prediction of incident diabetes mellitus by baseline IGF1 levels
}

\author{
Harald Jörn Schneider*, Nele Friedrich ${ }^{1, *}$, Jens Klotsche ${ }^{2}$, Sabine Schipf ${ }^{3}$, Matthias Nauck ${ }^{1}$, Henry Völzke ${ }^{3}$, \\ Caroline Sievers ${ }^{4}$, Lars Pieper ${ }^{2}$, Winfried März ${ }^{5}$, Hans-Ulrich Wittchen ${ }^{2}$, Günter Karl Stalla ${ }^{4, *}$ and \\ Henri Wallaschofski ${ }^{1, *}$ \\ Medizinische Klinik-Innenstadt, Ludwig-Maximilians University, Ziemssenstrasse 1, 80336 Munich, Germany, ${ }^{1}$ Institute of Clinical Chemistry and \\ Laboratory Medicine, University of Greifswald, 17475 Greifswald, Germany, ${ }^{2}$ Institute of Clinical Psychology and Psychotherapy, Technical University \\ Dresden, Dresden, Germany, ${ }^{3}$ Institute for Community Medicine, University of Greifswald, 17475 Greifswald, Germany, ${ }^{4}$ Max Planck Institute of \\ Psychiatry, 80804 Munich, Germany and ${ }^{5}$ Synlab Medizinisches Versorgungszentrum Heidelberg GmbH, 69214 Eppelheim, Germany \\ (Correspondence should be addressed to H J Schneider; Email: harald.schneider@med.uni-muenchen.de)
}

*(H J Schneider, N Friedrich, G K Stalla and H Wallaschofski contributed equally to this work)

\begin{abstract}
Objective: IGF1 is associated with metabolic parameters and involved in glucose metabolism. Low-IGF1 has been implicated in the etiology of glucose intolerance and subjects with pathological causes of either low- or high-IGF1 are at risk of diabetes. We hypothesized that both low- and high-IGF1 levels increase the risk of diabetes and aimed to assess the role of IGF1 in the risk of developing diabetes in a large prospective study.

Design: An analysis of two prospective cohort studies, the DETECT study and SHIP.

Methods: We measured IGF1 levels in 7777 nondiabetic subjects and assessed incident diabetes mellitus during follow-up.

Results: There were 464 cases of incident diabetes during 32229 person-years (time of follow-up in the DETECT study and SHIP: 4.5 and 5 years respectively). There was no heterogeneity between both studies $(P>0.4)$. The hazard ratios (HRs) of incident diabetes in subjects with IGF1 levels below the 10th or above the 90th age- and sex-specific percentile, compared to subjects with intermediate IGF1 levels, were 1.44 (95\% confidence interval (CI) 1.07-1.94) and 1.55 (95\% CI 1.06-2.06) respectively, after multiple adjustment. After further adjustment for metabolic parameters, the HR for low-IGF1 became insignificant. Analysis of IGF1 quintiles revealed a U-shaped association of IGF1 with risk of diabetes. Results remained similar after exclusion of patients with onset of new diabetes within 1 year or with borderline glucose or HbA1c levels at baseline.

Conclusions: Subjects with low- or high-IGF1 level are at increased risk of developing diabetes.
\end{abstract}

European Journal of Endocrinology 164 223-229

\section{Introduction}

Diabetes mellitus is a major cause of morbidity and death worldwide. The main causes of diabetes known today are obesity and related consequences, such as insulin resistance, hypertension, dyslipidemia, and inflammation (1-4). Experimental evidence suggests that insulin-like growth factor 1 (IGF1) counteracts these mechanisms at various levels $(5,6)$.

However, clinical data to support this evidence are scarce. Smaller studies suggest that low-IGF1 or a genetic polymorphism causing low-IGF1 level is associated with increased risk of glucose intolerance or diabetes $(7,8)$ and patients with low-IGF1 in GH deficiency have an increased prevalence of metabolic syndrome (9).

On the other hand, acromegalics with high-IGF1 due to GH excess are at high risk of diabetes (10). Correspondingly, we found increased prevalence of diabetes in subjects with both low- and high-IGF1 in a cross-sectional study (11).

Therefore, we hypothesized that subjects from the general population with either low- or high-IGF1 are at increased risk of diabetes. To test this hypothesis, we assessed the incidence of diabetes in nondiabetic subjects as a function of IGF1 in two cohorts, namely, the Diabetes Cardiovascular Risk-Evaluation: Targets and Essential Data for Commitment of Treatment (DETECT) study and the Study of Health in Pomerania (SHIP).

\section{Subjects and methods}

\section{Subjects}

Both studies conformed to the principles of the Declaration of Helsinki and were approved by the local ethics committees. All subjects gave written informed 
consent. The SHIP only included subjects of Caucasian origin. In the DETECT study, we did not record ethnicity, but, being representative of the German population, the participants were mainly of Caucasian ethnicity.

In the DETECT study, all information was collected by the treating physicians. The baseline evaluation was performed on one of two specified half-days in September 2003 (12). All patients were invited to a follow-up visit at 1 year and at 4.5 years. Diabetes was recorded at baseline, at 1-year-follow-up visit in 2004 and at 4.5-year follow-up visit between September 2007 and February 2008.

In the SHIP, baseline data were collected from 1997 through 2001 (13). All participants were invited to a follow-up visit at 5 years. Diabetes was recorded at baseline and during the 5-year follow-up from 2003 to 2006. Figure 1 shows the flowchart of subjects included in this study. Altogether, the total study population comprised 7777 subjects.

\section{Instruments and measures}

Blood was sampled and medical information was collected as described previously $(9,14)$. Subjects were either fasting or non-fasting in the DETECT study and non-fasting in the SHIP.

In both the studies IGF1 was determined only at baseline with an automated chemiluminescence system (Nichols Institute Diagnostics, San Clemente, CA, USA). The maximal intra- and inter-assay coefficients of variation were 5 and $7 \%$ respectively. Glucose was

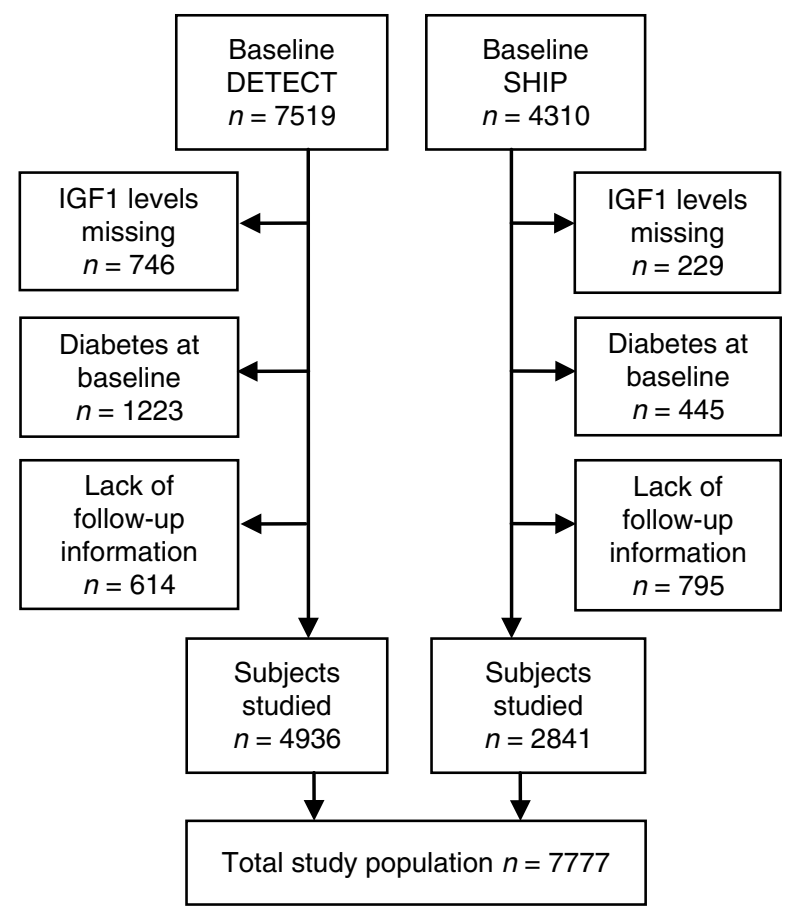

Figure 1 Flowchart of subjects included. determined enzymatically (Roche Diagnostics). HbAlc was determined chromatographically (ADAMS HA 8160 analyzing system and Recipe Chemicals + Instruments GmbH in the DETECT study and SHIP respectively).

We defined diabetes mellitus both at baseline and at follow-up as self-reported history of diabetes, intake of oral antidiabetic drugs or insulin, or $\mathrm{HbA1c}$ levels $\geq 6.5 \%$ (15). Hypertension and dyslipidemia were defined as described previously (11).

\section{Statistical analysis}

We calculated age- and sex-specific percentiles of IGF 1 using 5-year strata of age. In a power calculation, assuming a 1.5-fold increased risk of incident diabetes in subjects with IGF1 levels below the 10th or above the 90th percentile, we calculated a power of 0.814 , of 0.670 , and 0.971 for the DETECT study, SHIP, and both studies combined.

Discrete survival time models (16) were calculated with defined survival times at 1-year and 4.5-year follow-ups in the DETECT study and 5-year follow-up in the SHIP. Complementary log-log regression models (17) were performed in the person $\times$ year data to estimate the hazard ratios (HRs) of incident diabetes as a function of baseline IGF1.

Besides crude analyses (model 1), we adjusted for the following covariates: model 2 - age, sex, and study; model 3 - model $2+$ smoking status, education, family status, physical activity, and alcohol consumption; model 4 - model 3 +hypertension, glucose, dyslipidemia, and waist-to-height ratio.

For sensitivity analyses, we assessed whether the effects persisted after exclusion of the first year of follow-up or after exclusion of patients with borderline glucose (non-fasting glucose $>140 \mathrm{mg} / \mathrm{dl}$ ) or $\mathrm{HbAlc}$ (6.0-6.5\%) at baseline.

All statistical analyses were performed using STATA 10.1. A value of $P<0.05$ was considered statistically significant. The S.E.M. and confidence intervals (CIs) of parameter estimates were calculated by the HuberWhite-Sandwich (18) matrix to account for the clustered sampling design.

\section{Results \\ Baseline characteristics}

Table 1 displays the selected baseline demographic and clinical characteristics of the subjects included in this study. Among the classes of IGF1, there were differences in education, physical activity, alcohol consumption, hypertension, $\mathrm{HbAlc}$, and measures of obesity in at least one of the two cohorts. Table 2 displays the differences between subjects lost to follow-up and the subjects included in this study. 


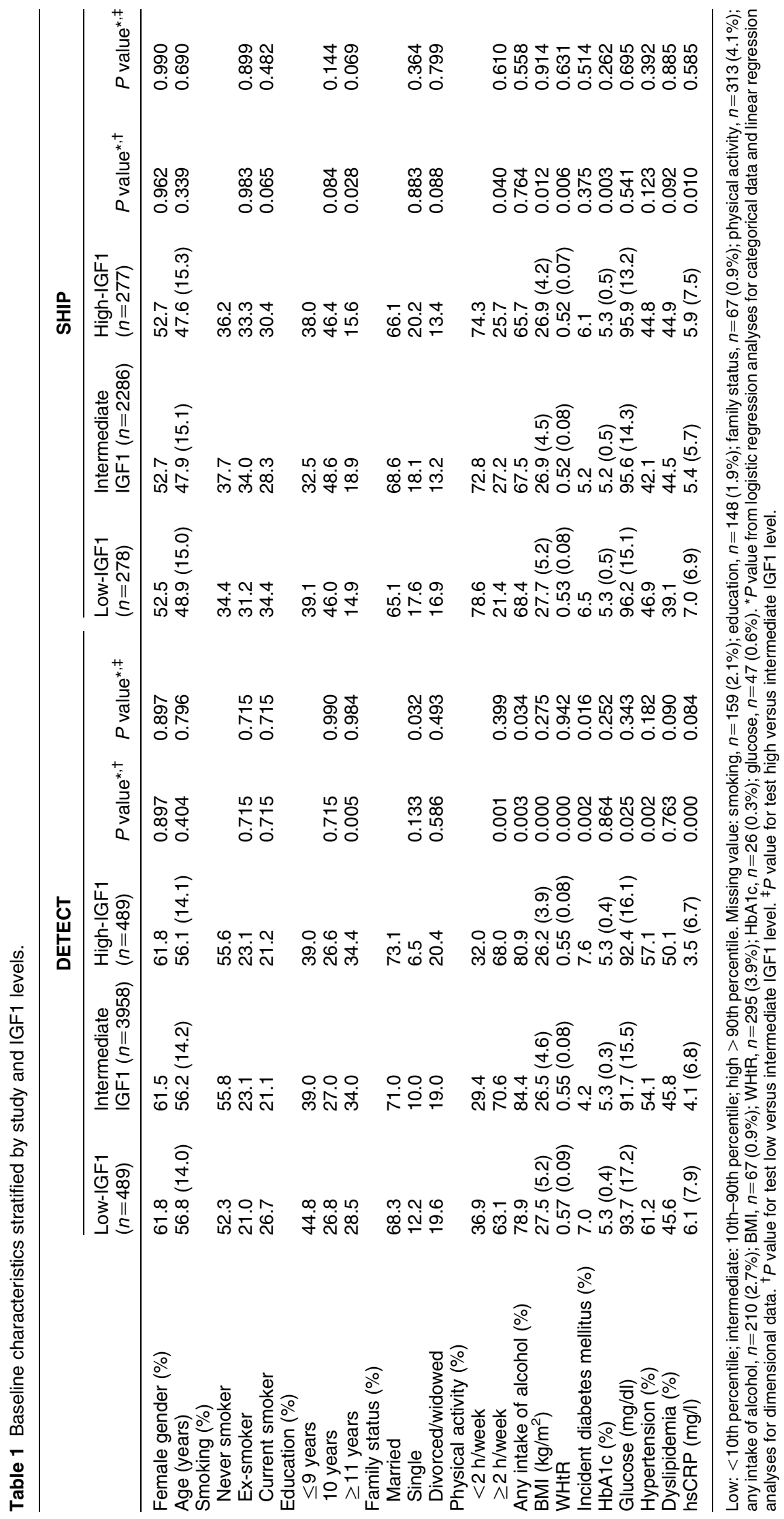


Table 2 Baseline characteristics of subjects lost to follow-up and subjects included in the study.

\begin{tabular}{|c|c|c|c|}
\hline & $\begin{array}{c}\text { Study } \\
\text { population } \\
(n=7777)\end{array}$ & $\begin{array}{c}\text { Dropout } \\
(n=1406)\end{array}$ & $\boldsymbol{P}$ value* \\
\hline Female gender (\%) & 58.3 & 53.3 & 0.000 \\
\hline Age (years) & $51.1(18.1)$ & $53.2(15.1)$ & 0.000 \\
\hline IGF1 level & $138.6(53.5)$ & $144.7(61.7)$ & 0.001 \\
\hline Low-IGF1 (\%) & 10.4 & 9.6 & 0.061 \\
\hline Intermediate IGF1 (\%) & 80.1 & 80.8 & \\
\hline High-IGF1 (\%) & 9.5 & 9.6 & \\
\hline \multicolumn{4}{|l|}{ Smoking (\%) } \\
\hline Never smoker & 48.6 & 37.9 & 0.016 \\
\hline Ex-smoker & 26.9 & 25.3 & \\
\hline Current smoker & 24.5 & 36.8 & \\
\hline \multicolumn{4}{|l|}{ Education (\%) } \\
\hline$\leq 9$ years & 37.4 & 45.2 & 0.000 \\
\hline 10 years & 34.8 & 32.1 & \\
\hline$\geq 11$ years & 27.8 & 22.8 & \\
\hline \multicolumn{4}{|l|}{ Family status (\%) } \\
\hline Married & 69.9 & 56.2 & 0.000 \\
\hline Single & 13.0 & 22.0 & \\
\hline Divorced/widowed & 17.1 & 21.3 & \\
\hline \multicolumn{4}{|l|}{ Physical activity (\%) } \\
\hline$<2$ h/week & 53.2 & 41.5 & 0.000 \\
\hline$\geq 2 \mathrm{~h} /$ week & 46.8 & 58.5 & \\
\hline Any intake of alcohol (\%) & 77.6 & 69.5 & 0.000 \\
\hline $\mathrm{BMI}\left(\mathrm{kg} / \mathrm{m}^{2}\right)$ & $26.8(4.9)$ & $26.7(4.6)$ & 0.563 \\
\hline WHtR & $0.54(0.09)$ & $0.54(0.08)$ & 0.448 \\
\hline $\mathrm{HbA1c}(\%)$ & $5.3(0.5)$ & $5.3(0.4)$ & 0.173 \\
\hline Glucose $(\mathrm{mg} / \mathrm{dl})$ & $93.9(15.2)$ & $93.3(15.2)$ & 0.196 \\
\hline Hypertension (\%) & 50.6 & 47.3 & 0.041 \\
\hline Dyslipidemia (\%) & 45.4 & 44.3 & 0.375 \\
\hline hsCRP (mg/l) & $5.6(7.5)$ & $4.5(6.9)$ & 0.028 \\
\hline
\end{tabular}

Low: <10th percentile; intermediate: 10th-90th percentile; high $>90$ th percentile. BMI, body mass index; $\mathrm{WHtR}$, waist to height ratio. ${ }^{\star} P$ value from logistic regression analyses for categorical data and linear regression analyses for dimensional data.

\section{Associations between IGF1 and incident diabetes}

In the DETECT study and SHIP, 310 and 154 subjects respectively, developed diabetes during follow-up (464 pooled cases during 32229 person-years). The incidence rate was 14.4 per 1000 person-years.

In the DETECT study, the crude HRs for incident diabetes below the 10th and above the 90th percentile of IGF1 were 1.60 (95\% CI 1.16-2.20) and 1.44 (95\% CI 1.03-2.01) respectively. In the SHIP, the crude HRs pointed to the same direction but were weaker and not significant (1.25 (95\% CI 0.76-2.06) and 1.18 (95\% CI $0.71-1.97)$ respectively). The $\chi^{2}$ test for homogeneity (19) revealed no heterogeneity among studies $(P=0.400$ and 0.509 respectively). Since the power for the SHIP was too low and we found no heterogeneity, we decided to pool the data from both studies.

Table 3 displays the association of IGF1 with diabetes. High IGF1 was consistently associated with increased risk of diabetes after full adjustment. Low IGF1 was associated with increased risk of diabetes after adjustment for multiple covariates, but this association became weaker and insignificant after further adjustment for hypertension, glucose, dyslipidemia, and waist-to-height ratio. Additional adjustment for C-reactive protein did not change the results (data not shown). IGF1 used as a continuous quadratic term remained a significant and independent predictor after full adjustment. The results were similar for men and women after separate analysis by sex (data not shown). Analysis of IGF1 quintiles revealed a U-shaped association with the lowest risk of future diabetes in the 3rd quintile of IGF1 (Fig. 2).

\section{Sensitivity analyses}

To address the problem of reverse causation (the possibility that precursors of diabetes influence IGF1 levels rather than IGF1 levels being causally related to the development of diabetes), we assessed whether the effects persisted after exclusion of the first year of followup (only in the DETECT study since 1-year follow-up was not available in the SHIP). In another analysis, we excluded patients with borderline glucose (non-fasting glucose $>140 \mathrm{mg} / \mathrm{dl})$ or HbAlc $(6.0-6.5 \%)$ at baseline in the pooled studies. Even though type 1 diabetes is unlikely to occur in the adult age, we ran an additional sensitivity analysis by exclusion of subjects aged $<35$ years, to rule out type 1 diabetes with certainty. Table 3 displays the results. The effect estimates remained similar in the sensitivity analyses and the significances remained unchanged.

\section{Discussion}

The main finding of our study is a U-shaped association of IGF1 levels with risk of incident diabetes. This association was persistent after multiple adjustments even though the strength of the effect was weak to moderate. The association of low IGF1 with incident diabetes became weaker and insignificant after further adjustment for abdominal obesity, hypertension, glucose, and dyslipidemia.

We cannot rule out that low IGF1 levels are truly confounded by these metabolic markers, i.e. that these markers influence both IGF1 levels and diabetes risk. However, it is also possible that these markers are in the causal pathway of low IGF1 and diabetes.

Evidence suggests a reciprocal association between low IGF 1 levels and metabolic syndrome. On one hand, there is evidence that IGF1 protects against production of free fatty acids, systemic inflammation, $\beta$-cell dysfunction, insulin resistance, and hypertension and, thus, low IGF1 promotes metabolic syndrome and development of diabetes $(5,6)$. On the other hand, the GH-IGF1 axis is blunted in obese subjects and partially restored after weight loss intervention such as bariatric surgery (20), possibly suggesting an opposite causality. 


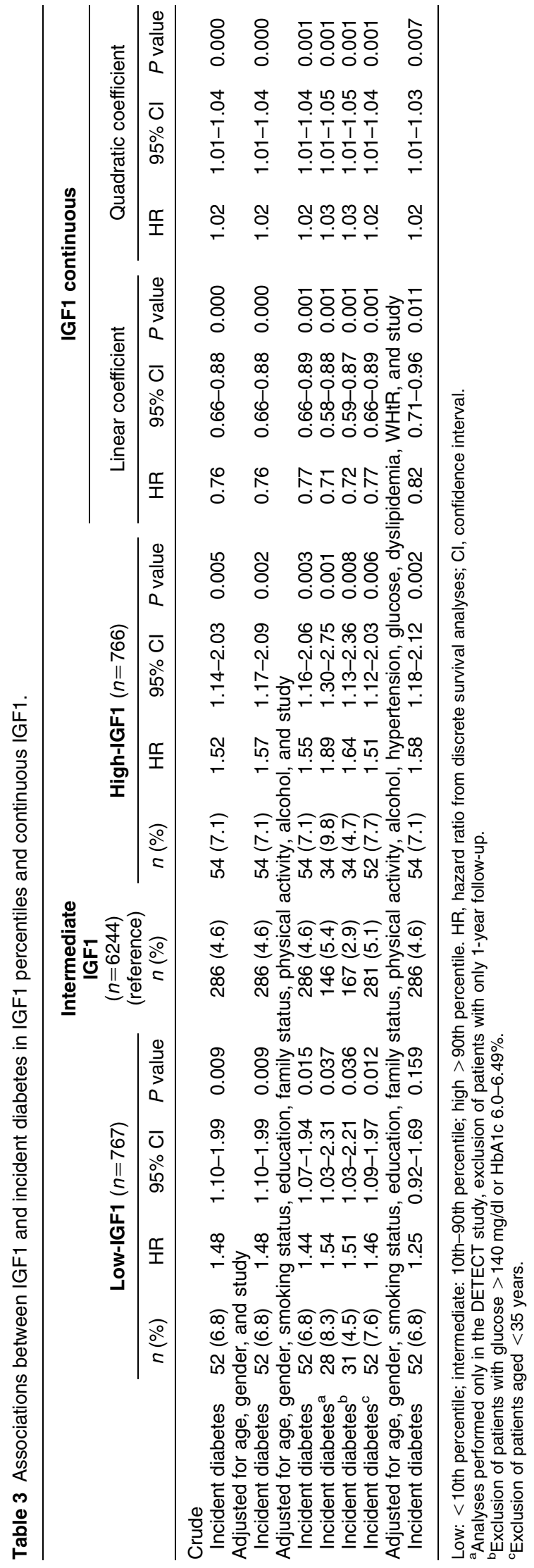

IGF 1 is mainly bound to IGF-binding proteins (IGFBPs-1 to -6) and other plasma proteins. IGFBPs protect IGF1 from rapid degradation and regulate its bioavailability (6). Moreover, IGFBPs are modulated by insulin. IGFBP-1 correlates positively with insulin sensitivity and high-IGFBP-2 suggests high-insulin sensitivity and might be increased by insulin (21). Thus, IGF1 levels in this study also reflect the complex interplay between insulin, glucose metabolism, and the IGF system.

There is no clear explanation for the association of high-IGF1 level with increased risk of diabetes. GH stimulates IGF1 secretion and decreases insulin sensitivity (22). In transgenic mice, overexpressing IGF1, hypoglycemia, and resistance to diabetes were found (23). However, mice overexpressing GH have high-IGF1, insulin resistance, and increased likelihood of developing diabetes (24).

Potentially, though speculative, in subjects with high-IGF1, GH secretion is increased causing insulin resistance and diabetes. In fact, we have found the prevalence of acromegaly to be higher than expected in the DETECT study (25). Yet, most subjects with abnormally high-IGF1 level did not have pathologically increased GH secretion. Therefore, if increased GH is the cause of elevated diabetes risk, the increase must be subclinical in most cases. Thus, the pathomechanisms involved in the association of IGF1 with the development of diabetes are likely to be different for low and high levels of IGF1: although a lack of protective effects against diabetes seems to play a role in low-IGF1 state, potentially increased GH secretion or other, unknown, causes might be involved in states of high-IGF1.

This study confirms and extends the findings of Sandhu et al. (7) and of Vaessen et al. (8). They have found an increased risk of glucose intolerance or diabetes in subjects with low-IGF1 level or a genetic polymorphism associated with low-IGF1 level respectively. However, unlike this study, those studies have not found a U-shaped association with increased risk of diabetes at both low- and high-IGF1 levels. Potential reasons for these differences include differences in study size or design and mere chance. Both studies were significantly smaller than our studies and had fewer events. Moreover, Vaessen et al. (8) used a dichotomized predictor, not allowing assessing curvilinear associations. In addition, the findings of Vaessen et al. (8) were not confirmed by another study (26).

This study has several strengths and limitations. A major strength is the prospective design, the size, and large number of events. This allows making assumptions on causality and detecting weak to moderate effects. Furthermore, the fact that the same method was used for IGF1 measurement and there was no heterogeneity between the studies also allowed pooling the two cohorts.

We did not measure insulin, homeostatic model assessment (HOMA) index, GH secretion, or IGFBPs. Thus, we were not able to further analyze the 


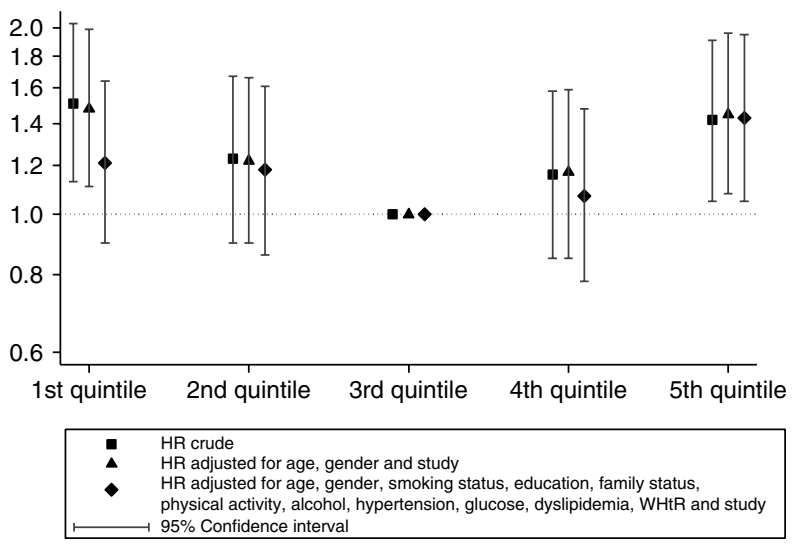

Figure $2 \mathrm{U}$-shaped association of incident diabetes mellitus and IGF1.

associations between these systems. This study was mainly designed to identify whether IGF1 levels can be used as biomarkers for diabetes risk rather than to elucidate the complex interplay of associated factors. Further studies, assessing the factors mentioned above, are needed to answer the question how these factors interact with IGF1 and diabetes.

Like in any cohort study, we cannot rule out residual confounding. Moreover, the number of events is still too limited for a detailed subgroup analysis. The follow-up time might be too short to detect late effects. In addition, we cannot exclude that the dropouts from this study were informative. To address this issue, we compared the dropouts and completers at baseline. Even though some differences were significant, these differences were generally small. Therefore, we think a potential bias due to dropout is likely to be small.

We cannot rule out reverse causation with certainty. However, to address this problem we excluded subjects with incident diabetes within 1 year or with borderline glucose or $\mathrm{HbA1c}$. This did not change or even increased the effect estimates. Therefore, reverse causation appears unlikely and IGF1 seems to play a true causal role in the development of diabetes.

This study was performed in a mainly Caucasian, European population, representative of the German population. We do not know whether our findings are generalizable to other populations or ethnicities.

In summary, we have shown that subjects with lowor high-IGF1 level are at increased risk of developing diabetes. IGF1 is an independent predictor of diabetes. Therefore, IGF1 might improve risk stratification for incidence of diabetes, if our results are confirmed and validated in further studies. Though our study did not allow elucidating the underlying pathomechanisms, it seems likely that different mechanisms are involved in the associations of low- and high-IGF1 with risk of diabetes. Further studies are needed to confirm these findings, to test generalizability, and to elucidate the underlying pathomechanisms.

\section{Declaration of interest}

The authors declare that there is no conflict of interest that could be perceived as prejudicing the impartiality of the research reported.

\section{Funding}

The Diabetes Cardiovascular Risk-Evaluation: Targets and Essential Data for Commitment of Treatment (DETECT) is a cross-sectional and prospective, longitudinal, nationwide clinical epidemiological study. The DETECT was supported by an unrestricted educational grant from Pfizer GmbH, Karlsruhe, Germany. Members of the DETECT study group include: principal investigator: Prof. Dr H-U Wittchen; staff members: Dipl.-Psych. L Pieper, Dipl.-Math. J Klotsche, Dr T Eichler, Dr H Glaesmer, E Katze. Steering Committee: Prof. Dr H Lehnert (Lübeck), Prof. Dr G K Stalla (München), Prof. Dr A M Zeiher (Frankfurt); advisory board: Prof. Dr W März (Heidelberg/Graz), Prof. Dr S Silber (München), Prof. Dr U Koch (Hamburg), Priv.-Doz. Dr D Pittrow (München/Dresden), Prof. Dr M Wehling (Mannheim), Dr D Leistner (Frankfurt), Priv.-Doz. Dr H J Schneider (München), Dr C Sievers (München). The SHIP is part of the Community Medicine Research net (CMR) of the University of Greifswald, Germany, which was funded by the Federal Ministry of Education and Research, the Ministry of Cultural Affairs as well as the Social Ministry of the Federal State of Mecklenburg-West Pomerania. The CMR encompasses several research projects, which share data from the population-based Study of Health in Pomerania (SHIP; http://ship. community-medicine.de). Statistical analyses were supported by the Competence Network Diabetes of the German Federal Ministry of Education and Research.

\section{References}

1 Stern MP, Williams K \& Haffner SM. Identification of persons at high risk for type 2 diabetes mellitus: do we need the oral glucose tolerance test? Annals of Internal Medicine 2002136 575-581.

2 Thorand B, Löwel H, Schneider A, Kolb H, Meisinger C, Fröhlich M \& Koenig W. C-reactive protein as a predictor for incident diabetes mellitus among middle-aged men: results from the MONICA Augsburg Cohort Study, 1984-1998. Archives of Internal Medicine 2003 163 93-99. (doi:10.1001/archinte.163.1.93)

3 Schulze MB, Hoffmann K, Boeing H, Linseisen J, Rohrmann S, Möhlig M, Pfeiffer AF, Spranger J, Thamer C, Häring HU, Fritsche A \& Joost HG. An accurate risk score based on anthropometric, dietary, and lifestyle factors to predict the development of type 2 diabetes. Diabetes Care 200730 510-515. (doi:10.2337/dc062089)

4 Wilson PW, Meigs JB, Sullivan L, Fox CS, Nathan DM \& D'Agostino RB Sr. Prediction of incident diabetes mellitus in middle-aged adults: the Framingham Offspring Study. Archives of Internal Medicine 2007167 1068-1074. (doi:10.1001/archinte. 167.10.1068)

5 Rajpathak SN, Gunter MJ, Wylie-Rosett J, Ho GY, Kaplan RC, Muzumdar R, Rohan TE \& Strickler HD. The role of insulin-like growth factor-I and its binding proteins in glucose homeostasis and type 2 diabetes. Diabetes/Metabolism Research and Reviews 200925 3-12. (doi:10.1002/dmrr.919)

6 Ezzat VA, Duncan ER, Wheatcroft SB \& Kearney MT. The role of IGF-I and its binding proteins in the development of type 2 diabetes and cardiovascular disease. Diabetes, Obesity and Metabolism 2008 10 198-211. (doi:10.1111/j.1463-1326.2007.00709.x)

7 Sandhu MS, Heald AH, Gibson JM, Cruickshank JK, Dunger DB \& Wareham NJ. Circulating concentrations of insulin-like growth factor-I and development of glucose intolerance: a prospective observational study. Lancet 2002359 1740-1745. (doi:10.1016/ S0140-6736(02)08655-5)

8 Vaessen N, Heutink P, Janssen JA, Witteman JC, Testers L, Hofman A, Lamberts SW, Oostra BA, Pols HA \& van Duijn CM. 
A polymorphism in the gene for IGF-I: functional properties and risk for type 2 diabetes and myocardial infarction. Diabetes 2001 50 637-642. (doi:10.2337/diabetes.50.3.637)

9 van der Klaauw AA, Biermasz NR, Feskens EJ, Bos MB, Smit JW, Roelfsema F, Corssmit EP, Pijl H, Romijn JA \& Pereira AM. The prevalence of the metabolic syndrome is increased in patients with GH deficiency, irrespective of long-term substitution with recombinant human GH. European Journal of Endocrinology 2007 156 455-462. (doi:10.1530/EJE-06-0699)

10 Melmed S. Medical progress: acromegaly. New England Journal of Medicine 2006355 2558-2573. (doi:10.1056/NEJMra062453)

11 Schneider HJ, Klotsche J, Saller B, Lehnert H, Böhler S, Sievers C, Pittrow D, Ruf G, März W, Erwa W, Zeiher AM, Silber S, Wittchen HU \& Stalla GK. Associations of age-dependent insulin-like growth factor-1 standard deviation scores to diseases and risk conditions: cross-sectional study in 6,282 primary care patients. European Journal of Endocrinology 2008158 153-161. (doi:10.1530/EJE-07-0600)

12 Wittchen HU, Glaesmer H, März W, Stalla GK, Lehnert H, Zeiher AM, Silber S, Koch U, Böhler S, Pittrow D \& Ruf G. Cardiovascular risk factors in primary care: methods and baseline prevalence rates - the DETECT program. Current Medical Research and Opinion 200521 619-629. (doi:10.1185/030079905X38187)

13 John U, Greiner B, Hensel E, Lüdemann J, Piek M, Sauer S, Adam C, Born G, Alte D, Greiser E, Haertel U, Hense HW, Haerting J, Willich S \& Kessler C. Study of Health in Pomerania (SHIP): a health examination survey in an east German region: objectives and design. Social and Preventive Medicine 200146 186-194. (doi:10.1007/BF01324255)

14 Friedrich N, Haring R, Nauck M, Lüdemann J, Rosskopf D, SpilckeLiss E, Felix SB, Dörr M, Brabant G, Völzke H \& Wallaschofski H. Mortality and serum insulin-like growth factor (IGF)-I and IGF binding protein 3 concentrations. Journal of Clinical Endocrinology and Metabolism 2009 94 1732-1739. (doi:10.1210/jc.2008-2138)

15 International Expert Committee. International Expert Committee report on the role of the $\mathrm{A} 1 \mathrm{C}$ assay in the diagnosis of diabetes. Diabetes Care 200932 1327-1334. (doi:10.2337/dc09-9033)

16 Singer JD \& Willett JB. It's about time: using discrete-time survival analysis to study duration and the timing of events. Journal of Educational Statistics $1993 \mathbf{1 8} 155-195$. (doi:10.2307/1165085)

17 Clayton D \& Hills M. Statistical Models in Epidemiology, pp 1-375 Oxford: Oxford University Press, 1993.

18 Royall RM. Model robust confidence intervals using maximum likelihood estimators. International Statistical Review $1986 \mathbf{5 4}$ 221-226. (doi:10.2307/1403146)
19 Grennland S \& Robins JM. Estimation of a common effect parameter from sparse follow-up data. Biometrics $1985 \mathbf{4 1}$ 55-68. (doi:10.2307/2530643)

20 Edén Engström B, Burman P, Holdstock C, Ohrvall M, Sundbom M \& Karlsson FA. Effects of gastric bypass on the GH/IGF-I axis in severe obesity - and a comparison with GH deficiency. European Journal of Endocrinology 2006154 53-59. (doi:10.1530/eje.1. 02069)

21 Arafat AM, Weickert MO, Frystyk J, Spranger J, Schöfl C, Möhlig M $\&$ Pfeiffer AF. The role of insulin-like growth factor (IGF) binding protein-2 in the insulin-mediated decrease in IGF-I bioactivity. Journal of Clinical Endocrinology and Metabolism $2009 \mathbf{9 4}$ 5093-5101. (doi:10.1210/jc.2009-0875)

22 Møller N \& Jørgensen JO. Effects of growth hormone on glucose, lipid, and protein metabolism in human subjects. Endocrine Reviews 200930 152-177. (doi:10.1210/er.2008-0027)

23 Robertson K, Lu Y, De Jesus K, Li B, Su Q, Lund PK \& Liu JL. A general and islet cell-enriched overexpression of IGF-I results in normal islet cell growth, hypoglycemia, and significant resistance to experimental diabetes. American Journal of Physiology. Endocrinology and Metabolism 2008294 E928-E938. (doi:10. 1152/ajpendo.00606.2007)

24 Olsson B, Bohlooly-Y M, Fitzgerald SM, Frick F, Ljungberg A, Ahrén B, Törnell J, Bergström G \& Oscarsson J. Bovine growth hormone transgenic mice are resistant to diet-induced obesity but develop hyperphagia, dyslipidemia, and diabetes on a highfat diet. Endocrinology 2005146 920-930. (doi:10.1210/en. 2004-1232)

25 Schneider HJ, Sievers C, Saller B, Wittchen HU \& Stalla GK. High prevalence of biochemical acromegaly in primary care patients with elevated insulin-like growth factor-1 levels. Clinical Endocrinology 200869 432-435. (doi:10.1111/j.1365-2265. 2008.03221.x)

26 Frayling TM, Hattersley AT, McCarthy A, Holly J, Mitchell SM, Gloyn AL, Owen K, Davies D, Smith GD \& Ben-Shlomo Y. A putative functional polymorphism in the IGF-I gene: association studies with type 2 diabetes, adult height, glucose tolerance, and fetal growth in U.K. populations. Diabetes $2002512313-2316$. (doi:10.2337/diabetes.51.7.2313)

Received 2 November 2010

Accepted 8 November 2010 